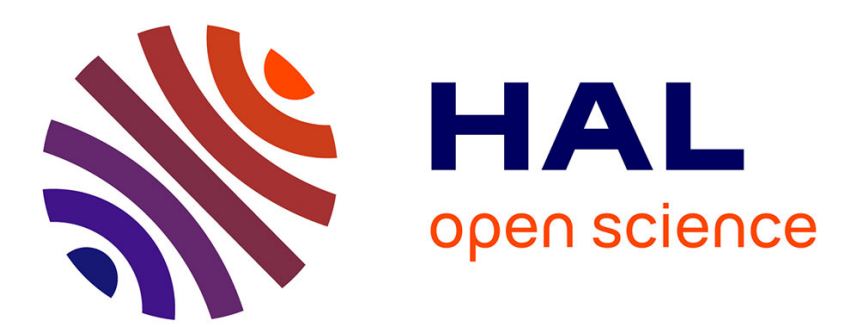

\title{
Méthode instrumentée pour l'amélioration continue des processus logistiques
}

\author{
Matthieu Lauras, Matthieu Dupuy, Jacques Lamothe
}

\section{To cite this version:}

Matthieu Lauras, Matthieu Dupuy, Jacques Lamothe. Méthode instrumentée pour l'amélioration continue des processus logistiques. Journal Européen des Systèmes Automatisés (JESA), 2007, 41 (3-4), pp.333 - 364. 10.3166/jesa.41.333-364 . hal-01783150

\section{HAL Id: hal-01783150 \\ https://hal.science/hal-01783150}

Submitted on 6 Nov 2018

HAL is a multi-disciplinary open access archive for the deposit and dissemination of scientific research documents, whether they are published or not. The documents may come from teaching and research institutions in France or abroad, or from public or private research centers.
L'archive ouverte pluridisciplinaire HAL, est destinée au dépôt et à la diffusion de documents scientifiques de niveau recherche, publiés ou non, émanant des établissements d'enseignement et de recherche français ou étrangers, des laboratoires publics ou privés. 


\title{
Méthode instrumentée pour l'amélioration continue des processus logistiques
}

\author{
Matthieu Lauras $^{1}$ - Matthieu Dupuy $y^{1,2}$ - Jacques Lamothe ${ }^{1}$ \\ ${ }^{1}$ Centre Génie Industriel - \\ Ecole des Mines d'Albi-Carmaux \\ ${ }^{2}$ Direction de la Logistique - \\ Campus Jarlard, route de Teillet \\ Pierre Fabre SA \\ 81009 Albi-France \\ 8 rue Christian d'Espic \\ matthieu.lauras@enstimac.fr, \\ 81106 Castres - France \\ matthieu.dupuy@pierre-fabre.com
}

jacques.lamothe@enstimac.fr

RÉSUMÉ. De nombreux dirigeants d'entreprises considèrent aujourd'hui la gestion de leurs chaînes logistiques comme une priorité. L'enjeu consiste à optimiser simultanément la satisfaction de leurs clients et la rentabilité nécessaire de leurs activités. Mais l'instabilité récurrente des marchés les oblige à repenser de façon permanente leurs processus au travers d'une démarche d'amélioration continue. Un rapide tour d'horizon des méthodes et outils relatifs à chaque étape de cette démarche souligne deux limites pour le cas des processus logistiques. D'abord, il ne semble pas exister de réel consensus quant à la méthode à adopter pour structurer la phase d'observation d'un système logistique. Nous proposons alors un référentiel de diagnostic des chaînes logistiques permettant de dégager les caractères discriminants d'un système logistique. Ensuite, notre analyse met à jour un manque d'outils permettant de faire le lien entre cette phase de diagnostic (et notamment l'étape de modélisation) et la phase de traitement de la démarche d'amélioration. Nous proposons pour ce faire une grille instrumentée d'analyse / actions permettant d'établir ce lien. Les deux propositions sont déclinées sur le cas d'une chaîne logistique pharmaceutique.

ABSTRACT. Managers regard the management of their supply chains as a priority. The objective consists in simultaneously optimizing the customer satisfaction and the necessary profitability of their activities. But the market instability forces them to permanently consider their processes through a continuous improvement step. A rapid review of the methods and tools relating to each stage of this step underlines two limits for the logistic processes. Firstly, it does not seem to exist a consensus as for the method to adopt to structure the observation phase of a logistic system. We then propose a supply chain framework of diagnosis allowing to release the discriminating characters of a logistic system. Then, our analysis updates a lack of tools making it possible to establish the link between this phase of diagnosis (and in particular the modelling stage) and the treatment phase of the improvement step. We propose with this intention an instrumented grid of analysis/actions allowing to establish this bond. The two proposals are declined on the case of a pharmaceutical supply chain.

MOTS-CLÉS : Amélioration continue, chaînes logistiques, processus, diagnostic.

KEYWORDS: Continuous improvement, supply chains, processes, diagnosis. 


\section{Introduction}

L'amélioration continue et les chaînes logistiques constituent une dynamique dont tout le monde se réclame et que certains recherchent. Car au-delà du simple soucis de l'image de l'entreprise, la compétition mondiale oblige à innover, diversifier, réduire les coûts, améliorer la qualité et livrer de plus en plus vite.

Les chaînes logistiques partagent ces objectifs. Ce sont des systèmes de soustraitants, de producteurs, de distributeurs, de détaillants et de clients entre lesquels s'échangent les flux matériels dans le sens des fournisseurs vers les clients et des flux d'information dans les deux sens [Tayur et al, 1999]. Elles œuvrent à l'atteinte d'un optimum global: la satisfaction du client - qui n'est d'ailleurs pas nécessairement la somme d'optima locaux. Plusieurs entreprises participent à de telles chaînes, pour un produit / service, une gamme de produits / services, ou un secteur économique. La gestion de ces chaînes logistiques (ou Supply Chain Management - SCM) visent notamment à optimiser les processus qui les animent. En ce sens, elles portent à un niveau supérieur - celui de la filière de production - les initiatives passées d'optimisation de la production au sein d'une entreprise, tout en les amplifiant. Les démarches d'amélioration continue ont donc toute leur place dans l'optimisation de la gestion de ces chaînes logistiques.

L'amélioration continue d'un système vise principalement à améliorer les processus mis en oeuvre. Une illustration commune est de changer les opérations individuelles des exécutants pour rendre leur travail plus productif, moins fatiguant et plus sûr. Dans le contexte d'une chaîne logistique, par nature complexe, une démarche d'amélioration continue se doit d'être contrôlée. Il est inconcevable que n'importe qui puisse «bricoler» les processus de façon autonome, sous prétexte d'amélioration. Plusieurs méthodes proposent alors d'organiser la démarche d'amélioration continue autour de quatre grandes étapes (PETRA [Berrah et al., 2001], GIM [Doumeingts et Vallespir, 2004], le six sigma [Eckes, 2001], SAF [Kettinger et al., 1997] et bien sûr les normes ISO 9000 v2000) :

- Observer le système étudié pour collecter l'information utile et commencer à appréhender les modes de fonctionnement et les caractéristiques intrinsèques dudit système ;

- Modéliser le système étudié, sur la base des éléments collectés précédemment, pour décrire l'organisation et les processus d'un système dans le but de les analyser et de les restructurer ;

- Analyser cette représentation du système pour en dégager des axes de progrès potentiels ;

- Traiter les dysfonctionnements avec des méthodes de re-engineering (au sens de l'amélioration de la performance des systèmes industriels). 
L'intégralité de la démarche (cf. Figure 1) est exécutée en parallèle d'une phase d'évaluation qui, spécifie à chaque étape les niveaux de performance du système. Il s'agit de permettre l'analyse de l'existant (performance réelle), d'aider à la définition des marges d'amélioration (position vis-à-vis de référentiels externes) et de valider l'impact des actions de progrès mises en œuvre (mesures de performance a posteriori).

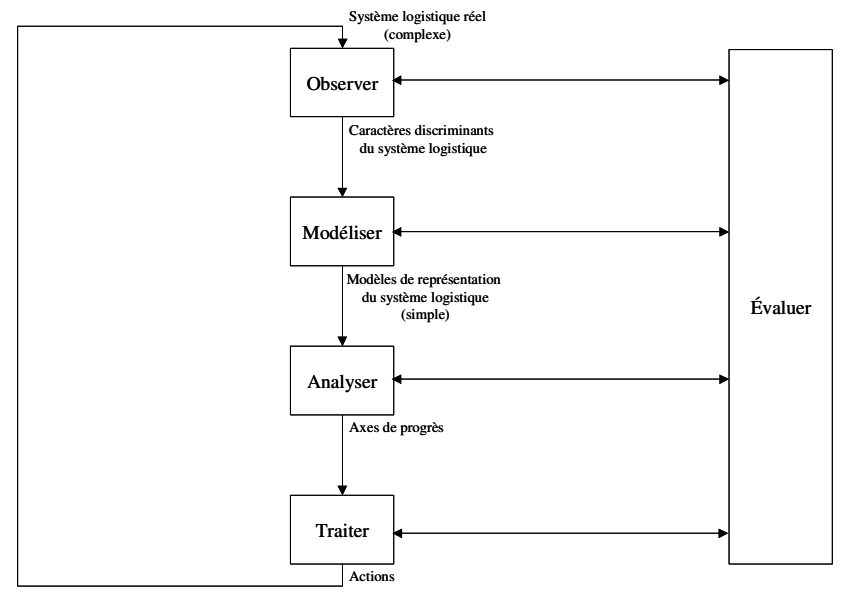

Figure 1 : Les quatre étapes de la démarche d'amélioration continue.

La déclinaison d'une telle démarche sur un système de la taille d'une chaîne logistique pose alors le problème de l'appréhension, de la compréhension, de l'interprétation et de l'évolution des processus logistiques.

La partie 2 aborde cette question en présentant les principaux outils et méthodes qui permettent d'instrumenter chacune des phases de la démarche dans le cadre de processus logistiques. Deux points durs sont identifiés concernant les phases d'observation et d'analyse.

La partie 3 développe une proposition de référentiel de diagnostic pour les chaînes logistiques permettant de révéler les caractères discriminants de tout système logistique (instrument pour la phase observer).

La partie 4 présente une méthode pratique d'analyse pour faire le lien entre un modèle de représentation d'un système logistique et des actions de re-engineering à mettre en œuvre (instrument pour la phase analyser).

Les deux propositions sont déclinées sur le cas d'une chaîne logistique pharmaceutique. 


\section{Quels outils et méthodes pour une démarche d'amélioration continue dans les chaînes logistiques ?}

Dans cette partie, nous développons chaque étape de la démarche afin d'identifier les outils et méthodes susceptibles de l'instrumenter.

\subsection{Observer : structurer l'enquête en logistique}

Le besoin de constater quels sont les modes de fonctionnement de la chaîne logistique suppose par essence l'utilisation d'un outil de collecte et de formalisation de données. Or, l'observation directe est longue, impossible si l'on veut faire un travail à grande échelle. On se tourne alors naturellement vers une méthode d'enquête, l'idée étant de questionner les acteurs pour avoir des indications sur les différents facteurs et sur les tendances générales. Les supports d'enquêtes dans le domaine de la logistique restent aujourd'hui très confidentiels. Il existe néanmoins des référentiels disponibles (moyennant finances) sur le sujet, généralistes ou métiers. Ces référentiels constituent davantage des supports d'audit que de diagnostic. On entend par-là qu'ils cherchent à évaluer l'exécution et le respect d'engagements et de bonnes pratiques établis en amont (cahier des charges, normes Qualité, etc.). Le diagnostic, lui, suppose une approche des états et modes de fonctionnement existant dans la chaîne logistique sans se référer à un référentiel prédéfini. On citera comme exemple de référentiels logistiques d'audit :

- la formation et le guide d'audit logistique de l'ASLOG (Association Française pour la Logistique) : évaluation des pratiques de la chaîne logistique [ASLOG, 2003] ;

- l'évaluation logistique EVALOG : renforcement de la relation client/fournisseur dans le milieu de l'automobile [Rouquet, 2004] ;

- $\quad$ et bien sûr, dans une certaine mesure, les normes ISO 9000 - v2000 qui définissent un système logistique et qualité et associent un référentiel d'audit [AFNOR, 2000].

Dans tous les cas, les objectifs de ces démarches et documents portent sur l'analyse des processus clés et la mesure de performance de l'organisation en prenant en compte la dimension transversale de la logistique de façon à pouvoir anticiper les mutations éventuelles vers de nouvelles organisations.

Toutes ces approches insistent sur la nécessité d'analyser toutes les fonctions de la chaîne logistique depuis l'approvisionnement jusqu'à la distribution des produits (et même leur reprise). Elles soulignent toutes, par ailleurs, l'aspect fondamental des fonctions transverses (planification notamment) et des fonctions de relation aux clients comme par exemple le marketing et la vente. 
En conclusion, il n'existe pas, à proprement parler, de référentiel d'élaboration d'un questionnaire d'analyse de système logistique. Pourtant, il s'agit bien là de l'élément central d'une intervention visant à établir la photographie exacte d'une chaîne logistique et des composantes coopératives qui la composent. L'objet de la partie 3 est justement de proposer un tel référentiel.

\subsection{Modéliser : exploiter les méthodes de modélisation d'entreprise}

La modélisation d'entreprise permet de structurer la connaissance que l'on a d'une entreprise pour permettre a posteriori son analyse [Vernadat, 1999]. Elles constituent par essence l'instrument de référence de la phase de modélisation de la démarche d'amélioration continue de processus logistiques.

\subsubsection{Les différents concepts de la modélisation d'entreprise}

\subsubsection{Les cadres de modélisation}

Un cadre de modélisation est «une structure de modèles partiels reposant sur des dimensions, au sein duquel on chemine afin de comprendre le tout »[Pingaud, 2005]. Il permet ainsi de réduire la complexité de la modélisation d'entreprise en proposant divers éléments de classification.

Parmi les cadres de modélisation existant pour la modélisation d'entreprise, nous pouvons citer GIM, GERAM et surtout la norme européenne ENV 40003 «Framework for Enterprise Modelling» qui définit les concepts génériques nécessaires pour la modélisation d'entreprise. Les trois dimensions de l'architecture selon cette norme sont :

- Niveau de modélisation : l'élément de plus haut niveau est le périmètre du système étudié et la stratégie associée (identification et conceptualisation) puis la spécification des besoins, la conception, l'implémentation, la réalisation et le démantèlement.

- Niveau de généricité : générique, partiel et spécifique.

- Points de vue : fonctionnel pour formaliser les processus opérationnels, informationnel pour décrire les objets de l'entreprise ; ressource pour la description des moyens nécessaires à la mise en œuvre des processus ; et organisationnel pour identifier les rôles et responsabilités.

Pour être complet, il convient d'ajouter un point de vue qui n'apparaît pas explicitement dans la norme : le point de vue décisionnel. Il est en effet intégré dans chacune des vues de la norme (fonctionnelle et organisationnelle notamment) sans pour autant disposer d'une vue à part entière. 


\subsubsection{Panorama des méthodologies et modèles existants}

Une méthodologie est un ensemble de méthodes qui contribue à résoudre un problème en les utilisant selon des règles établies [Pingaud, 2005]. Pour la modélisation d'entreprise, nous pouvons citer GRAI, PETRA, MECI, ACNOS, OLYMPIOS, OSSAD, SAF...

Un modèle est une représentation d'une abstraction de la réalité exprimée dans un langage [Pingaud, 2005]. Pour chacun des points de vue du cadre de référence plusieurs modèles sont disponibles [Lauras, 2004][Dupuy, 2005] :

- $\quad$ Point de vue fonctionnel : IDEF0 ou SADT, IDEF2 et IDEF3 sont les modèles de représentation des processus les plus connus. D'autres modèles existent comme les diagrammes d'activités d'UML, les modèles conceptuels de traitement de MERISE, les réseaux de Pétri ou encore les BPMN (Business Process Modeling Notation).

- Point de vue informationnel : les formalismes les plus employés sont ici les diagrammes entités - associations, IDEF1 / IDEF1x et les diagrammes de classes d'UML.

- Point de vue ressource : nous pouvons retenir ici les réseaux de Pétri, le Graphcet et le Statechart qui permettent de représenter la dynamique des ressources et le modèle entités - associations qui spécifient les aptitudes et compétences proposées par les ressources.

- Point de vue organisationnel : les modèles les plus utilisés sont ici les organigrammes.

- $\quad$ Point de vue décisionnel : le modèle de référence est GRAI.

Au final, il apparaît clairement que la phase de modélisation d'une démarche d'amélioration continue de processus logistiques peut s'appuyer sur une palette très fournie et très complète de méthodes issues de la modélisation d'entreprise. S'il est évident que ces méthodes facilitent la compréhension du système étudié, il convient néanmoins de s'interroger sur l'exploitation qui peut en être faite lors de la phase d'analyse pour identifier des axes de progrès potentiels et surtout pour faire le lien avec les outils de re-engineering à mettre en œuvre.

\subsection{Analyser : structurer une approche de la caractérisation à l'action}

La phase d'analyse telle qu'elle est considérée ici cherche à exploiter les modèles d'entreprise issus de la phase précédente pour identifier les axes de progrès à exploiter.

Ici encore, il existe de nombreuses méthodes dites d'analyse. La plupart d'entre elles sont dédiées à des problématiques de conception ou d'ateliers de production et 
mettent l'accent sur la Qualité. On citera [Dupuy, 2005] par exemple les méthodes d'analyses :

- fonctionnelles : analyse de la valeur, process maping, value stream maping, cahier des charges fonctionnel, etc.

- fonctionnelles et ressources : AMDEC, Audit, Benchmarking, cartes de contrôle, cercles de qualité, diagnostic d'anomalies / état machines, diagrammes de décision, diagrammes de Pareto, mesure des temps, diagrammes de dispersion, analyses factorielles, etc.

- ressources: diagrammes en arêtes de poisson, 5M, mesure de la capabilité, outils du fiabiliste (MTTF, MTBF...), etc.

Il faut noter que toutes ces méthodes d'analyse cherchent à identifier les causes de dysfonctionnement d'un système précis. En d'autres termes, les méthodes d'analyse présentés ici ont pour objet l'identification des causes racines à un problème particulier et non l'identification des axes d'amélioration au sein d'un système complexe. En ce sens, elles ne répondent pas à l'objectif d'amélioration continue des processus logistiques qui est le notre. En outre ces méthodes n'offrent pas de grilles de lecture permettant de passer facilement de l'analyse à l'outil de reengineering adéquat [Dupuy, 2005].

Au total, il semble que les méthodes d'analyse existantes n'offrent que peu de moyens de dégager des pistes de progrès dans le cadre d'une démarche d'amélioration continue appliquée aux processus d'une chaîne logistique. L'objet de la partie 4 est justement de proposer une grille d'analyse / action instrumentée pour faire le lien entre l'étape de diagnostic (observation et modélisation) et l'étape de traitement d'une démarche d'amélioration continue.

\subsection{Traiter : exploiter les outils et méthodes de re-engineering}

La phase de traitement de la démarche d'amélioration des systèmes industriels bénéficie de nombreux outils supports. Les actions associées à ces méthodes privilégient différentes orientations [Dupuy, 2005].

On trouve d'abord les démarches centrées sur l'élimination du gaspillage avec des méthodes comme la production au plus juste (Lean Manufacturing), des méthodes d'amélioration progressive au quotidien (Kaizen).

Les démarches centrées sur la réduction des défauts complètent ce tableau avec notamment des méthodes comme le management par la qualité totale (TQM) ou le six sigma (réduction de la variabilité à tous les niveaux).

Des démarches centrées sur le rendement des ressources apportent une gamme supplémentaire d'outils. Les principales méthodes qui supporte ces démarches sont le changement rapide d'outils (SMED) et la maintenance productive (TPM). 
Enfin, il existe une démarche centrée sur les évolutions radicales : le Business Process Reengineering.

Si ce panorama ne se veut bien sûr pas exhaustif, il souligne à quel point l'offre en termes de traitement des dysfonctionnements d'un système est vaste et complète.

Il semble donc que la phase de traitement de la démarche d'amélioration continue des processus logistiques puisse s'appuyer sur des méthodes et outils connus et disponibles.

\subsection{Evaluer : piloter la démarche d'amélioration}

L'ensemble des phases de la démarche que nous discutons dans ce papier s'appuie naturellement sur des outils d'évaluation de performance. Le pilotage de la démarche d'amélioration continue des processus logistiques suppose une évaluation qui ne se limite pas à la notion de mesure. Il s'agit d'identifier les actions à conduire pour mettre en œuvre les marges de progrès [Burlat et al., 2003]. Il faut disposer à la fois d'une vision de l'état du système et des leviers d'actions qui permettent de le faire évoluer. De nombreux outils et méthodes existent pour aider à la définition de systèmes d'indicateurs de performance qui soit pertinents : Activity Based Costing et Activity Based Management, Balanced Scorecard, ECOGRAI, modèle SCOR...

Il existe donc de nombreuses méthodes pour mettre en place un tableau de bord permettant de supporter la démarche visée d'amélioration continue.

\subsection{Synthèse de la problématique}

Le rapide tour d'horizon que nous venons de présenter sur les outils et méthodes associés aux différentes étapes de la démarche d'amélioration continue pour les processus logistiques souligne deux points durs.

D'abord, un manque de référentiel de diagnostic dédié aux chaînes logistiques permettant de garantir la pertinence de la phase d'observation. Nous proposons en partie 3 un référentiel pour répondre à ce point.

Ensuite, une absence d'outil permettant l'identification d'axes de progrès à partir des modèles de représentation des systèmes logistiques étudiés. Nous proposons en partie 4 une grille instrumentée pour répondre à ce point.

La figure ci-après présente sous forme de synoptique la problématique que nous traitons dans ce papier. 


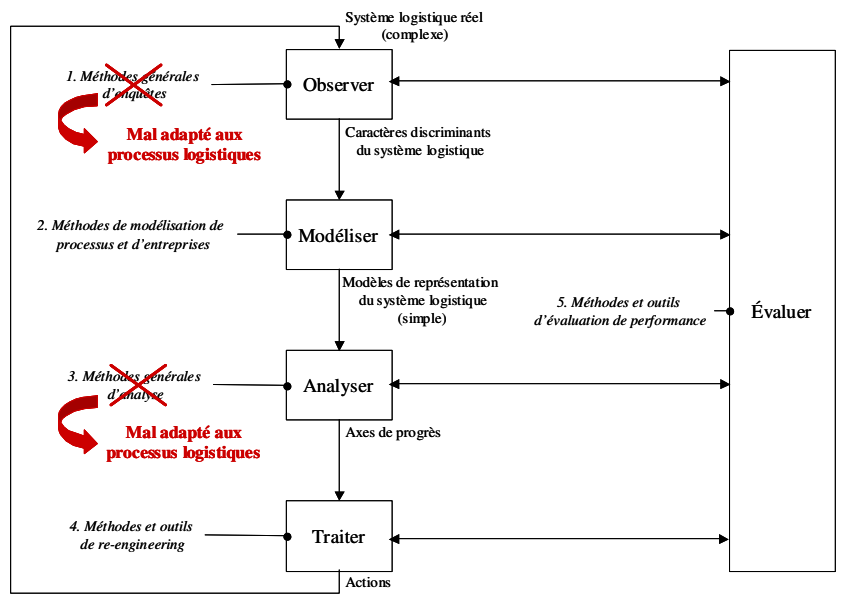

Figure 2 : Synoptique de la problématique

\section{Référentiel de diagnostic des processus des chaînes logistiques}

\subsection{Les fondements des chaînes logistiques}

Cette partie cherche à extraire des concepts fondamentaux des chaînes logistiques, les éléments qui structurent la gestion de ces chaînes.

\subsubsection{Du pilotage transversal des chaînes logistiques à la cartographie des flux}

Les différentes approches et définitions de la gestion des chaînes logistiques (SCM) [Mentzer et al., 2001][Stadtler, 2000][Arnold et Chapman, 2001] concordent toutes sur un point. Elles voient le $S C M$ comme une gestion coordonnée et surtout transversale des flux qui parcourent la chaîne logistique. Ces flux, rappelons-le, sont aussi bien de nature financière, informationnelle que physique. Ils constituent les objets qu'il convient de gérer dans le cadre des chaînes logistiques. La gestion des chaînes logistiques nécessite donc d'avoir une vision globale de ses flux pour pouvoir être en mesure de la piloter judicieusement. Il s'agit là d'une condition nécessaire, mais non suffisante, au SCM.

Cet axe d'analyse montre qu'un référentiel de diagnostic des systèmes logistiques doit disposer d'une composante dédiée à l'établissement d'une cartographie d'ensemble des flux de la chaîne logistique étudiée ; les flux à considérer étant les flux informationnels, physiques et financiers.

Cet axe d'analyse qualifie, en quelque sorte, les objets à gérer dans une chaîne logistique (« Quoi ? »). 
3.1.2 Des modèles de représentation des chaînes logistiques aux définitions des fonctions clés

[Lauras et al., 2003] rappellent que la notion de chaîne logistique peut finalement se résumer à un enchaînement d'ententes entre acteurs - d'une ou plusieurs entreprises, du premier des fournisseurs jusqu'aux consommateurs - permettant de mettre à disposition des clients leurs produits ou services. Deux modèles principaux offrent une formalisation de cette organisation: le modèle SCOR [Supply Chain Council, 2000] et le modèle proposé par [Kearney, 1994]. Ces deux modèles indiquent clairement qu'une chaîne logistique est composée de plusieurs fonctions inter-reliées : l'Approvisionnement, la Fabrication, la Distribution, la Planification globale, le Marketing, la Vente. Bien sûr, il existe également une «fonction» d'organisation ou de direction de chacune des sociétés intervenant dans les chaînes logistiques (fonction structurelle issue de l'existence même de l'entité). En termes de positionnement, ces fonctions intègrent la logique de flux (approvisionnement, fabrication, distribution), la relation aux clients (vente et marketing) et le pilotage de l'activité (planification et organisation).

L'analyse de tout système logistique doit donc inclure une vision et une démarche fonctionnelles. Il faut donc intégrer au référentiel un découpage par fonction des différentes questions et analyses à conduire. Les fonctions élémentaires à considérer sont l'approvisionnement, la fabrication, la distribution, la vente, le marketing, la planification globale et l'organisation.

Cet axe d'analyse présente les principaux intervenants dans les chaînes logistiques («Qui ? ») et de leurs missions («Pourquoi ? » de leurs fonctions).

Dès lors, il faut préciser quelles sont les composantes clés à identifier dans chacune de ces fonctions pour conduire une analyse d'un système logistique et de ses composantes coopératives. Pour ce faire, nous proposons de retenir l'interprétation des piliers du SCM de [Stadtler, 2000] proposée par [Lauras, 2004]. Cette interprétation identifie deux fondamentaux à la gestion des chaînes logistiques. D'une part, la «coordination » du SCM qui comprend le pilotage des variables de décision de planification en s'appuyant sur des Technologies de l'Information et de la Communication et sur des orientations processus. D'autre part, «l'intégration » du $S C M$ qui se définit comme le pilotage de l'architecture coopérative du réseau.

\subsubsection{Les outils de « coordination » du SCM}

\subsubsection{Les processus}

Un processus est une activité qui utilise des ressources de manière à permettre la transformation d'éléments d'entrée en éléments de sortie [AFNOR, 2000]. [Hammer et Champy, 1993] précisent qu'un processus doit être perçu comme un ensemble d'activités, orienté vers la production d'un résultat représentant une valeur pour le client. Le processus est donc une succession d'activités. La notion de valeur pour le client renvoie nécessairement à celle de performance et nécessite donc la définition, 
en amont, d'une consigne sur chacune des activités qui définissent le processus. L'objectif est de contrôler la chaîne de valeur. L'idée directrice de cette composante du SCM est finalement d'indiquer dans quelle mesure chaque fonction est pilotée dans une dynamique processus. C'est-à-dire dans quelle mesure les différentes fonctions qui définissent la chaîne logistique ont une connaissance précise des activités qui les composent, permettant d'appuyer une démarche d'amélioration continue ciblée et judicieuse. [Morley, 2002] nomme cette démarche le management par processus. Elle indique qu'il s'agit de répondre aux exigences de la qualité totale, en faisant évoluer les processus de façon lente et continue. Le système est alors vu comme un ensemble de processus corrélés. L'intérêt de cette démarche passe par une cartographie - une représentation - des processus, et en particulier des interfaces entre processus.

Le référentiel de diagnostic des systèmes logistiques se doit donc d'intégrer une analyse complète des éléments qui définissent les processus qu'il contient. Ainsi faut-il identifier les activités qui définissent chaque fonction, ainsi que les entrées et sorties, les ressources et les consignes qui les composent.

Cet axe d'analyse apporte un éclairage particulier sur «Qui ? fait «Quoi ? , précise « Pourquoi ? » (objectif) et qualifie «Comment ? » et « Avec quoi ? ».

\subsubsection{Les Technologies de l'Information et de la Communication (TIC)}

Les TIC englobent toutes les techniques de collecte, de stockage, de traitement et de diffusion de données quelles qu'elles soient [Balantzian, 2002]. Les progrès enregistrés ces dernières décennies en termes d'outils informatiques et de télécommunications ont profondément bouleversé la gestion d'entreprises et par là même, la gestion des chaînes logistiques. Le nœud du problème se situe clairement au niveau de l'information manipulée, il convient donc de s'arrêter quelques instants sur cette notion. [Pingaud et L'hôte, 2004] parlent d'information pour une donnée utilisée dans une situation déterminée. Ils précisent alors plusieurs propriétés qu'il convient d'associer à l'information et donc aux TIC. D'abord, l'information possède un sens et la valeur portée par la variable fournit donc une indication très précise pour son utilisateur. Ensuite, la qualité de l'information est étroitement dépendante de la situation à laquelle elle est attachée, et toute évolution de la situation peut entraîner une actualisation de l'information.

L'analyse des TIC dans la gestion des systèmes logistiques suppose donc une étude sur trois aspects :

- l'état des systèmes d'information utilisés (matériels, logiciels, architecture...);

- $\quad$ la justesse des informations (puisque variables au cours du temps) ;

- $\quad$ la pertinence des informations et outils supports au regard des besoins. 
Cet axe d'analyse complète la vue processus sur les ressources («Avec quoi ? ») et les informations exploitées («Comment?»). Il apporte en outre des informations sur les livrables («Pourquoi ?»).

\subsubsection{La planification}

La planification cherche à permettre les prises de décision appropriées pour l'utilisation optimale des ressources nécessaires à la réalisation de la demande. La planification se décline sur plusieurs horizons de temps et selon plusieurs degrés de finesse dans l'analyse des besoins par rapport aux capacités. A chacun de ces niveaux est associée une étape d'analyse comparée des charges à réaliser avec les capacités disponibles. Ces étapes permettent d'appuyer les décisions en termes d'utilisation de ressources et constituent donc la «garantie » que les demandes assignées au système seront bien satisfaites. Le Manufacturing Ressource Planning (MRP II) décrit l'articulation globale d'une démarche de planification.

[Eymery, 1997] et [Arnold et Chapman, 2001], entre autres, notent plusieurs propriétés nécessaires à la mise en œuvre, dans de bonnes conditions, d'un processus de planification. D'abord, à chaque étape de la démarche MRP II, ils indiquent que les décideurs utilisent, à leur niveau, trois types d'information : les besoins à réaliser, les capacités disponibles et bien sûr les process utilisés (qui permettent de transcrire les besoins en termes de charges). On voit ici que ces informations sont à la base du processus décisionnel et doivent donc être fiables et suffisantes. Ils soulignent par ailleurs que l'évaluation, à chaque étape de la planification, de l'adéquation entre charges et capacités, suppose l'existence de règles d'arbitrage permettant, par exemple, d'anticiper la réalisation de certaines demandes et de réguler les ressources disponibles. Ces règles de gestion constituent clairement la première valeur ajoutée d'une telle démarche. Ils rappellent ensuite que les différentes étapes de la planification s'appuient toutes sur des outils, qu'ils soient formels ou non, structurés (Material Requirements Planning (MRP), Distribution Requirement Planning $(D R P) \ldots)$ ou pas. Enfin, ils insistent tous sur l'importance d'une gestion budgétaire cohérente. Il s'agit en effet d'alimenter dans un premier temps les réflexions quant aux possibilités - financières - offertes au système en termes de choix de gestion. Dans un deuxième temps, une gestion budgétaire réalisée en amont soutiendra les arbitrages faits (investissements, maîtrise des coûts, etc.).

Il faut donc intégrer au référentiel la description des modalités de gestion de la demande afin de situer chaque élément de la démarche globale MRP II. L'accent doit être mis également sur les éléments suivants : choix de gestion (règles d'arbitrages), nature et qualité des informations sources, méthodes et outils employés et enfin, typologie de gestion budgétaire utilisée.

En définitive, cet axe d'analyse permet de compléter le diagnostic en proposant une vision de l'organisation temporelle («Quand?») et spatiale («Où ?»). Il offre en outre des explications quant aux critères de choix («Comment ? ») et ressources utilisés («Avec quoi ?»). 
Nous devons, à ce stade, préciser un aspect important de la démarche de planification. $\mathrm{Si}$, comme nous venons de le montrer, la démarche de planification existe bien dans chacune des fonctions de l'entreprise (chaque fonction doit effectivement gérer une demande, une capacité et un process de réalisation), l'objet même de gestion des chaînes logistiques, imposant un pilotage transversal des opérations, suppose une centralisation des éléments de planification. C'est pourquoi, comme nous l'indiquions plus haut, il existe donc bien aussi une fonction, à part entière, dite de «planification globale». L'objet de cette partie consistait à développer la composante «planification» de chaque fonction de la chaîne logistique (Marketing, Vente, Distribution, Approvisionnement, Fabrication et Planification globale).

\subsubsection{Les outils « d'intégration »}

[Stadtler, 2000] identifie trois composantes essentielles aux relations d'ententes dans les chaînes logistiques : les partenaires, le pilotage du réseau ainsi constitué et les spécifications de ce dernier.

\subsubsection{Les partenaires}

Un partenaire est un acteur interne ou externe, individuel ou collectif, spécialisé ou généraliste qui intervient dans une entente industrielle quelle qu'elle soit. Un partenaire peut finalement prendre les traits tout aussi bien d'une filiale, d'un soustraitant, d'un fournisseur que d'un prestataire... [Poirier et Reiter, 2001] et [Stadtler, 2000] insistent sur le fait que le choix d'un partenaire doit être articulé autour de la satisfaction des besoins du client. Cette opération de sélection se base principalement sur l'analyse de la valeur ajoutée du partenaire potentiel par rapport aux compétences existantes dans la chaîne logistique. La caractérisation des candidats doit permettre de valider la correspondance entre des besoins exprimés d'une part, et les compétences, connaissances, moyens et savoir-faire proposés d'autre part. En outre, les besoins évoluant dans le temps (car les attentes des clients évoluent également), il est indispensable de disposer d'outils de suivi de l'adéquation des apports du partenaire aux dits besoins. Il faut bien remarquer que cette démarche de rapprochement ne se limite pas à de simples critères économiques et doit également intégrer l'ensemble des besoins d'ordre métier [Arnold et Chapman, 2001]. L'environnement des chaînes logistiques et des attentes des clients étant par nature instable, [Poirier et Reiter, 2001] soulignent enfin l'utilité d'intégrer dans la relation au partenaire, une démarche de progrès (projets, synergies, etc.).

Le diagnostic d'un système logistique et des ententes qui le composent doit nécessairement considérer une spécification des partenaires intervenants. La pertinence du choix de ces partenaires repose alors sur l'adéquation entre des besoins exprimés en relation avec les attentes des clients et des critères de choix d'ordre économique bien sûr, mais aussi métiers. Enfin, le contexte changeant des chaînes logistiques sous-tend la nécessité de garantir la fiabilité du partenariat et d'inscrire, dans le temps, une démarche de progrès. 
Cet axe d'analyse met finalement en avant «Avec qui ? » on fait «Quoi ? ». Il présente en outre le «Pourquoi ? » du choix des partenaires et «Comment? » cette relation s'inscrit dans la durée.

\subsubsection{Le pilotage du réseau}

Une chaîne logistique fait intervenir différentes entités qui ne sont pas forcément regroupées sous la même bannière juridique. Se pose alors la question de savoir comment est piloté le réseau ainsi constitué. Bien souvent, apparaît un leader naturel. Quatre raisons peuvent conduire à un tel choix [Stadtler, 2000]: le pouvoir financier, le meilleur - ou l'unique - savoir-faire (produits ou process), la plus grande valeur ajoutée sur l'ensemble de la chaîne, le fondateur de la chaîne logistique. Selon lui, ces causes suffisent à légitimer les décisions prises par cet acteur central auprès des autres intervenants de la chaîne logistique. Dans tous les autres cas, la prise de décision doit être organisée et le pouvoir partagé. Un comité de pilotage est alors constitué. Il est composé de représentants des acteurs de la chaîne logistique et est régi selon des règles précises. Ces règles sont soumises à négociation et doivent porter aussi bien sur le poids relatif des votes de chacun que sur le séquencement des interventions [Villarreal et al., 2004]. Par définition, une chaîne logistique fait intervenir plusieurs acteurs qui chacun dispose d'une histoire, d'une culture, de procédures et d'usages qui lui sont propres. Il convient d'intégrer ces éléments qui fixent, par essence, les degrés de liberté de la relation. En outre, le réseau coopératif s'intègre dans plusieurs activités régies par des lois, des réglementations ou encore des bonnes pratiques. Ces contraintes externes constituent bien évidemment des éléments structurants du pilotage des ententes qui composent la chaîne logistique. Les notions de pilotage et de performance sont indissociables. [Burlat et al., 2003] rappellent à ce sujet que «l'évaluation de performance n'est pas une fin en soi : elle n'est que l'un des maillons, certes fondamental, du processus de pilotage ». [Berrah et Haurat, 1997] précisent que pour piloter, il faut connaître à la fois sa position, la cible et les moyens dont on dispose pour le faire. Bref, le pilotage d'un réseau coopératif doit s'appuyer, comme n'importe quel système, sur une démarche d'évaluation de la performance.

Le pilotage d'un réseau coopératif se caractérise donc d'abord par la nature de sa structure de décision (leadership individuel ou collectif, niveau d'autonomie, règles d'arbitrage...). Ensuite, la vie d'un réseau doit intégrer les contraintes relatives à son environnement (réglementations, usages...). Enfin, qui dit pilotage, dit performance. Les ententes doivent donc être soumises à une réelle évaluation de performance (objectifs, variables d'action, moyens) et des outils doivent soutenir cette démarche.

Cet axe d'analyse souligne «Comment?» est piloté le réseau coopératif et insiste notamment sur les finalités de ce pilotage («Qui ? » et «Où ? ») et sur les moyens utilisés (« Avec Quoi ? »). 


\subsubsection{Nature et organisation du réseau coopératif}

Les travaux relatifs à la nature et à l'organisation des réseaux coopératifs ne sont pas légion et il est donc difficile de situer précisément les éléments qui structurent un réseau coopératif. Ce constat nous a conduit à proposer un référentiel de définition des différents états d'ententes industrielles [Lauras et al., 2003]. C'est donc sur la base de ce référentiel que nous allons orienter la structuration de l'axe d'analyse de la nature et des organisations en place dans les réseaux dits coopératifs. Cette proposition a montré que les ententes industrielles, quelles qu'elles soient, reposent sur quatre éléments fondamentaux : un objectif commun, le dépassement de limites individuelles, des modalités d'ententes et une gestion commune des aléas.

Concernant la notion d'objectif commun, il s'agit finalement de préciser quel est l'objet de l'entente, au-delà des simples intérêts individuels. Cet objectif peut être de différentes natures, selon que la mission dispose d'un caractère permanent ou conjoncturel. Ainsi, dans certains cas, il peut être utile de s'allier avec une autre compétence pour la réalisation d'un projet précis et donc d'un objectif unique. Dans d'autres cas, l'entente soutiendra une activité continue (fournisseur de matière première par exemple) et l'objectif associé sera sans doute soumis à une redéfinition fréquente (dynamique).

Les ententes visent également à apporter des solutions aux limites intrinsèques de chacun des intervenants [Burlat, 2002]. Pour cela, il faut être en mesure d'évaluer et de comparer aux possibilités individuelles - les besoins et les moyens utiles à l'atteinte de l'objectif global en termes de ressources (compétences, capacités...). Bref, il convient de cadrer quelles sont les forces et faiblesses de chaque acteur dans l'optique de la réalisation de l'objectif commun de façon à tisser des liens privilégiés sur des éléments ciblés.

Il existe de nombreuses formes de concrétisation, formelle ou non, d'une entente industrielle. Plusieurs questions se posent naturellement quant aux modalités qui définissent cette entente. S'agit-il d'un contrat? Quels sont les engagements et obligations des parties? Sur quel horizon et sur quel périmètre porte l'entente ? Quels sont les résultats escomptés ? etc. Autant d'éléments qui permettront de caractériser la nature des rapports coopératifs considérés.

Enfin, les ententes se doivent de gérer l'ensemble des aléas. Notre analyse du système logistique et de ses composantes coopératives doit donc intégrer cette dimension. Il s'agit notamment de situer les options prises pour anticiper les risques associés à l'opération, de noter les conséquences en cas de survenue et d'identifier les responsabilités [Sardas et al., 2002].

Ces quatre axes permettent de définir formellement le cadre organisationnel du réseau logistique coopératif étudié. A ce stade, il manque, pour être complet, une situation des différents états d'ententes au sein de ce réseau. Dans le cadre de la proposition de référentiel faite, il était indiqué que c'est la combinaison de deux 
facteurs qui caractérise les états d'ententes : les objets manipulés d'une part (données et/ou traitements), et la temporalité (échanges et/ou partages) d'autre part.

Le référentiel de diagnostic des systèmes logistiques et de leurs aspects coopératifs se doit finalement de considérer les éléments clés suivants pour caractériser la nature et l'organisation des ententes en place : les objectifs communs, les limites individuelles, les modalités de rapprochement (répartition des gains...), la gestion des aléas, la nature des objets manipulés, et la temporalité des interactions.

Ce dernier axe souligne le «Pourquoi?» des ententes en indiquant «Comment? » elles sont mises en œuvre. Il précise aussi les éléments («Quoi ?») et la temporalité («Quand?») qui les caractérisent.

\subsection{Proposition de structuration de référentiel pour l'observation des chaînes logistiques}

Dès lors, l'objet de la démarche vise à proposer une grille de référence qui facilite l'élaboration d'un questionnaire pour l'observation d'une chaîne logistique. Les combinaisons systèmes logistiques / objectif de l'intervention (et donc la formulation précise des questions) sont quasiment infinies. Ce qui ne permet pas de pouvoir définir une liste de questions qui soit générique et exhaustive. Par contre, la proposition portera sur la structuration (sections) du questionnaire et les principaux thèmes à traiter dans chacune des sections. Notre approche vise à répondre aux questions élémentaires d'un diagnostic quel qu'il soit : quoi ? quand ? où ? qui ? avec qui ? comment? avec quoi ? et pourquoi ? Nous l'avons vu précédemment, l'analyse d'un système logistique suppose d'abord un relevé des éléments de flux à gérer. Il s'agit ensuite, pour chacune des fonctions qui constituent la chaîne logistique, d'analyser d'une part les composantes liées aux modes de fonctionnement locaux et d'autre part, d'analyser la mise en œuvre des ententes industrielles sur lesquelles elles reposent. Notre proposition reprend ce constat et organise finalement le diagnostic en quatre temps (cf. figure suivante) :

- une cartographie transversale des flux: c'est le «quoi?» en termes d'objets manipulés ;

- un recueil des informations explicatives de la «coordination» de chaque fonction : c'est le quand? Où ? Qui ? Quoi ? Comment ? Avec quoi ? et pourquoi ? du fonctionnement local ;

- un recueil des informations explicatives de «l'intégration» de chaque fonction: c'est le quand? où ? qui ? quoi ? avec qui ? comment ? et pourquoi ? du fonctionnement des ententes ;

- une présentation de l'organisation générale du système : c'est le contexte. 


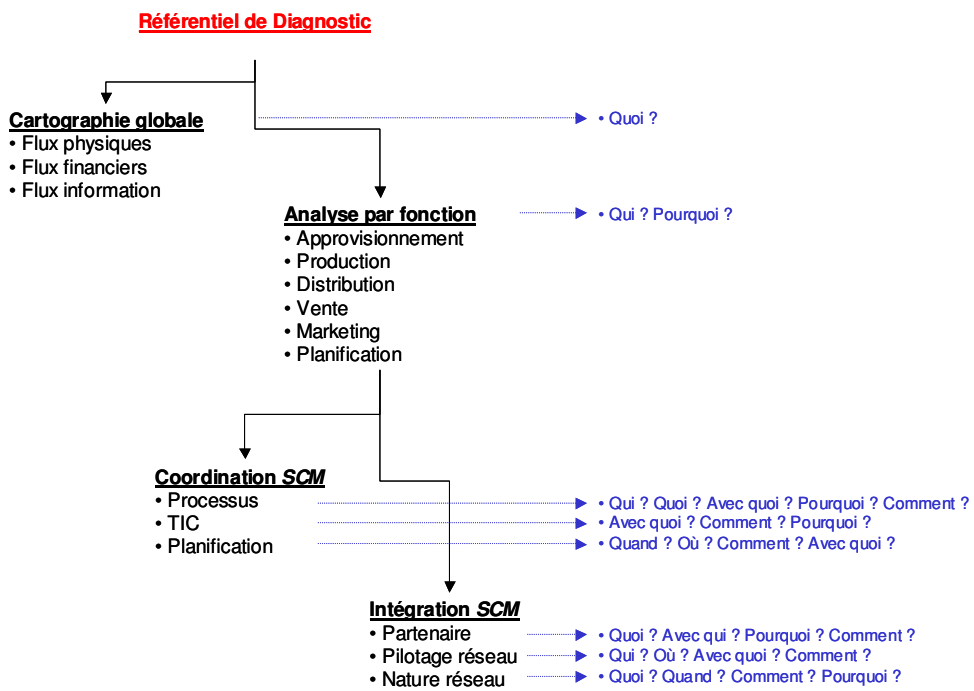

Figure - 3 : Architecture globale du référentiel de diagnostic logistique

\subsubsection{Diagnostiquer le «quoi ? » dans sa globalité}

Concernant les flux physiques, nous proposons de considérer trois catégories correspondant à la circulation logique des produits dans la chaîne. Ainsi, nous avons les flux amonts, les flux internes et les flux avals. Pour chacune de ces sections, il faut déterminer la composition des flux qui la parcourent. Nous proposons de travailler sur la base des éléments de volumes unitaires. Selon nous, il est possible de décomposer tout volume en cinq éléments :

- les volumétries traitées qui représentent l'aspect variable de l'activité ;

- les stocks qui sont une cible privilégiée de beaucoup de démarches d'amélioration du SCM ;

- le nombre de références produits manipulées qui impacte directement la complexité de gestion ;

- les effectifs de personnel qui représentent une part importante des coûts d'une chaîne logistique ;

- les partenaires (fournisseurs, sous-traitants, clients) pour lesquels des modes de gestion spécifiques sont mises en place et donc pour lesquels une connaissance des flux est indispensable.

Viennent ensuite les flux financiers. Établir une cartographie de ces flux repose sur deux concepts bien connus du contrôle de gestion et de la comptabilité. D'abord, le compte de résultats qui présente notamment la nature et les montants des recettes, dépenses et résultats. Ensuite, le bilan qui établit une photographie distincte des propriétés financières (actifs, actionnariat et dettes) du système étudié. Enfin, relativement à la cartographie des flux d'information, nous préconisons de considérer deux types d'axes. D'abord les flux statiques qui définissent les éléments 
fixes du système d'information. Les données stockées, les utilisateurs et les machines sont les différents flux statiques existants. Ensuite, nous considérons les flux informationnels dynamiques. Il s'agit simplement de la quantité d'information échangée d'une part, et des fréquences d'utilisation et de rafraîchissement de ces informations d'autre part.

3.2.2 Diagnostiquer le «quand? ", «où ? », « comment?», «qui ? », "quoi ?», "avec quoi? » et "pourquoi? » au niveau de chacun des acteurs de la chaîne logistique

Cette étude est conduite fonction par fonction. Il faut noter que bien que dans leur formulation finale, les questions sont différentes pour chaque fonction (les outils et processus utilisés ne sont pas les mêmes), la structuration du référentiel de diagnostic reste identique, quelle que soit la fonction considérée. Pour l'étude des modes de coordination, notre référentiel comprend trois grandes sections: l'orientation processus, les TIC et la planification.

Concernant l'orientation processus, nous avons vu qu'il était possible de décrire les processus opérationnels par l'intermédiaire de l'identification des activités pratiquées, des ressources et entrées utilisées, des sorties produites, ainsi que des objectifs assignés. Ces éléments s'accompagnent évidemment d'une démarche d'évaluation de la performance. Notre préconisation s'articule donc autour de ces cinq éléments.

Notre analyse des TIC a mis en évidence trois axes de différenciation: les logiciels et progiciels utilisés, la fiabilité des informations échangées et l'adéquation aux orientations stratégiques. Notre proposition s'appuie sur ces trois composantes de façon à mesurer les moyens mis en œuvre et leur pertinence eu égard aux besoins en termes d'échange et de partage d'information au niveau local.

Nous avons longuement insisté sur l'aspect central de la planification et sur son existence à tous les niveaux de décision et au sein de toutes les fonctions de la chaîne logistique. Nous avons notamment constaté que cette étape se caractérise par l'utilisation de méthodes et outils permettant de gérer la demande, sous condition budgétaire, sur la base de règles d'arbitrage et à partir d'informations sources (adéquation charge / capacité). Notre proposition reprend donc logiquement ces axes pour le diagnostic. 


\begin{tabular}{|c|c|c|}
\hline Sections & Sous-sections & Principaux Thèmes \\
\hline \multicolumn{3}{|c|}{ Coordination Logistique } \\
\hline \multicolumn{3}{|c|}{ Les Processus } \\
\hline & 1 Activités & Valeur ajoutée, opérations unitaires, temporalité... \\
\hline & 2 Ressources & Moyens humains, financiers, matériels... \\
\hline & 3 Entrées / Sorties & Eléments à traiter, résultats du processus, périodicité... \\
\hline & 4 Consignes & Objectifs de réalisation, lien avec la stratégie globale, périodicité... \\
\hline & 5 Performance locale & Tableaux de bord, objectifs, diffusion, complétude, intérêt... \\
\hline \multicolumn{3}{|l|}{ Les TIC } \\
\hline & 6 Systèmes d'information & Logiciels et progiciels, adéquation besoins, architecture... \\
\hline & 7 Fiabilité des informations & Mise à jour des données, documentation, justesse des utilisations... \\
\hline & 8 Adéquation aux orientations stratégiques & Adéquation aux besoins, limites, projets... \\
\hline & & \\
\hline & & \\
\hline & 10 Gestron ae ta aemanaet & 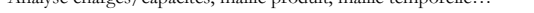 \\
\hline & 11 Métbodes et outils & Procédures, formulations, précisions... \\
\hline & 12 Informations sources & Exhaustivité, justesse, fraîcheur.. \\
\hline & 13 Choix de gestion & Priorités, leviers d'action, horizon.. \\
\hline
\end{tabular}

Tableau -1 Référentiel de diagnostic : coordination logistique pour une fonction

3.2.3 Diagnostiquer le «quand?», «où? », «combien?», «qui ? », «quoi ?», "avec qui ?", « avec quoi ? " et «pourquoi? » au niveau des ententes industrielles

Sur la même logique que précédemment, notre proposition consiste à décliner l'analyse que nous avons conduite sur les fondements des aspects coopératifs dans le cadre du SCM. Notre approche choisit d'intégrer la proposition de [Stadtler, 2000] et considère de ce fait trois axes pour situer les ententes (composantes coopératives) au sein d'une chaîne logistique : les partenaires, le pilotage du réseau et la nature même de ce réseau coopératif.

Relativement aux partenaires, qu'ils soient internes ou externes, nous avons mis en évidence plusieurs éléments caractéristiques et différenciants. Ainsi, les critères de choix et les spécifications d'un partenaire, les engagements mutuels pris et la démarche de progrès (intégrant le suivi de la performance) constituent les quatre sous-sections qu'il convient d'étudier pour établir un diagnostic pertinent de cette composante des ententes au sein des chaînes logistiques.

L'analyse que nous avons conduite plus haut au sujet du pilotage des réseaux coopératifs a montré la nécessaire considération de l'organisation du leadership, de l'existence de contraintes externes et de l'évaluation de la performance de l'entente.

Pour exploiter pratiquement l'axe de caractérisation de la nature des systèmes logistiques coopératifs, nous avons utilisé le référentiel défini par [Lauras et al., 2003] sur les ententes industrielles. Six items définissent les organisations en place en termes d'ententes. D'abord, la définition de l'objectif commun, le cadrage des limites individuelles, la formalisation des modalités de rapprochement et les conditions de la gestion commune des aléas définissent la nature même du réseau coopératif. Les objets manipulés (données et/ou traitements) et la temporalité des échanges et/ou partage précisent ensuite l'état d'entente. 


\begin{tabular}{|c|c|c|}
\hline Sections & Sous-sections & Principaux Thèmes \\
\hline \multicolumn{3}{|c|}{ Intégration Logistique } \\
\hline \multicolumn{3}{|c|}{$\begin{array}{c}\text { Les Partenaires } \\
\text { (internes et externes) }\end{array}$} \\
\hline & 14 Spécifications & Métier, localisation et implantation, image... \\
\hline & 15 Critêres de Choix & Qualité, coût, délai, politique, géographique... \\
\hline & 16 Expression de besoins et suivi & Engagements, tableaux de bord... \\
\hline & 17 Fiabilité et démarche de progrès & Suivi performance, projets, synergies... \\
\hline \multicolumn{3}{|l|}{ Le Pilotage } \\
\hline & 18 Leadership & Décideur (individuel, comité), niveau d'autonomie, régles d'arbitrage... \\
\hline & 19 Contraintes externes & Réglementations... \\
\hline & 20 Performance de l'entente & Tableaux de bord, objectifs, diffusion, complétude, intérêt... \\
\hline \multicolumn{3}{|c|}{ Le réseau coopératif } \\
\hline & 21 Objectifs communs & Définition, nature (structurel ou conjoncturel)... \\
\hline & 22 Limites individuelles & Compétences, ressources, capacités... \\
\hline & 23 Modalités de rapprocbement & Contractualisation, horizon, périmètre... \\
\hline & 24 Gestion des aleas & Anticipation des risques, évaluation des conséquences, responsabilités... \\
\hline & 25 Objets manipules & Données ou traitements (caractéristiques, complétudes, utilisation)... \\
\hline & 26 Temporalité & Echanges ou Partages, Fréquences de rafraîchissement... \\
\hline
\end{tabular}

Tableau -2 Référentiel de diagnostic : intégration logistique pour une fonction

\subsubsection{Situer l'organisation générale du système}

L'analyse de tout système (logistique ou pas) doit débuter par une prise de connaissance générale de celui-ci. C'est pourquoi nous rappelons ici qu'en plus des études ciblées décrites précédemment, il convient de ne pas omettre une phase de diagnostic de l'organisation générale permettant de connaître l'articulation globale.

\subsection{Application référentiel d'observation}

A partir du référentiel d'observation des chaînes logistiques proposé, nous avons pu caractériser la chaîne logistique intra-entreprise d'un groupe pharmaceutique. Nous présentons dans ce papier une illustration relative à une partie de la fonction de Production: les laboratoires de contrôle qualité. Le lecteur pourra se référer à [Lauras, 2004] et [Lauras et al., 2005] pour disposer d'autres illustrations sur les fonctions de Distribution, Approvisionnement, et Planification Globale.

La chaîne logistique étudiée est composée de plusieurs sites de production qui disposent chacune d'une unité de contrôle qualité indépendante des activités de production. La réglementation pharmaceutique impose en effet des contrôles à différents stades de la production (matières, composants, semis-finis, produits-finis, etc.). Le contrôle qualité est en interaction permanente avec d'autres maillons de la chaîne logistique comme la production, la distribution, l'administration des ventes ou la logistique.

Le contrôle qualité est une activité de service qui travaille à la commande pour la production, et qui produit une information et non un bien matériel. Sa charge ne peut pas être avancée. L'opération de contrôle qualité est donc comprise entre deux dates jalons (réception de l'échantillon et date de besoin). Puisque nous avions comme objectif au début de l'étude, l'amélioration du système «laboratoire de contrôle 
qualité », nous avons commencé par observer ce système à l'aide du référentiel que nous venons de présenter.

\subsubsection{Le questionnaire}

Une enquête préliminaire a été menée pour faciliter la formulation des supports d'interviews des laboratoires sur la base du référentiel de diagnostic. Cette phase a consisté en une série d'entretiens avec les services centraux qui entretiennent des relations fréquentes avec les laboratoires de contrôle : Planification industrielle, Assurance Qualité, Administration des Ventes, Distribution, Réglementaire, Logistique, Système d'Information. Nous avons pu structurer un questionnaire autour d'une cinquantaine de questions portant sur près de 80 thèmes du référentiel de diagnostic (une question peut effectivement renseigner plusieurs aspects de l'étude du système). Ces questions ont permis d'aborder l'ensemble des points que nous avions identifié dans le cadre de notre référentiel de diagnostic, dans la limite des coupes prédéfinies.

Pour des raisons de confidentialité évidentes, nous ne sommes pas en mesure de dévoiler ici le contenu exact de ces questions. Nous pouvons néanmoins en donner quelques illustrations :

- exemples de questions sur les Flux: Quelle est la quantité de dossiers traités (produits finis et semi-finis) ? Combien d'unités (masses, volumes, contenants...) expédiez-vous ? Combien d'utilisateurs informatiques gérez-vous ? Combien avez-vous de clients internes (par type) ? Etc.

- exemples de questions sur la Production: Comment planifiez-vous l'activité de l'analyse ? Quel est l'activité goulet ? Quel niveau de flexibilité et de polyvalence sur les ressources employées ? Quels sont vos délais de réalisation (cibles et constatés) ? Etc.

- exemples de questions sur la Planification: Comment établissez-vous vos prévisions de charge? Quels sont les règles de priorité dans la planification (type de produits, type de clients...) ? Quel tableau de bord avez-vous mis en place pour suivre votre activité ? Etc.

\subsubsection{La démarche d'enquête et ses résultats}

L'enquête (toujours en cours) concerne une dizaine de laboratoires laboratoires de contrôles du groupe (correspondants aux deux principales usines de production), pour acquérir une connaissance de la fonction, de son organisation et de ses modes de fonctionnement. Des missions sur sites par laboratoire ont été réalisées pour identifier de manière précise et exhaustive l'ensemble des données quantitatives d'une part (flux) et des données qualitatives d'autre part (coopération et intégration logistique de chaque activité). Pour ce faire, nous avons effectué des extractions informatiques des bases de données relatives aux dossiers de contrôle traités par chaque service de façon à préciser la cartographie exacte et détaillée des flux physiques et informationnels (dynamiques). Le complément des informations quantitatives (flux informationnels statiques et financiers) a été obtenu à la fois auprès des responsables de chaque unité. Relativement aux données qualitatives, 
nous avons conduit des interviews auprès des responsables de chaque activité de ce système logistique (Prélèvements, Ordonnancement, Analyses et saisie des résultats, Revue). Ces entretiens ont permis de remonter les informations relatives tant aux modes de fonctionnement locaux (processus, TIC et planification) qu'au niveau des aspects coopératifs avec les autres entités de la chaîne logistique (choix partenaire, pilotage de l'entente, nature et organisation de l'entente). Le taux de réponse à l'enquête a approché les $100 \%$. A partir des éléments recueillis, nous avons retenu une vingtaine de caractères explicatifs ${ }^{1}$ des processus de coordination, et une quinzaine de caractères explicatifs des processus d'intégration avec les autres entités de la chaîne logistique.

A titre d'illustration, nous pouvons présenter quelques caractères explicatifs que nous avons recensés dans le cadre de notre intervention :

- Relativement aux modes de fonctionnement locaux : mode de saisie des résultats (centralisé, par le technicien d'analyse), temps d'attente avant la mise en main (FIFO, LIFO, autre), typologie d'analyse (LCI, LCM, LCP), système de planification (planning mural, GPAO, rien), modalités de préparation des échantillons (centralisé, décentralisé), etc.

- Relativement aux ententes avec les autres entités : modes de transmission des dossiers de contrôle qualité (Système d'Information intégré, fax), stabilité des fournisseurs de composants (+, -), modalités de communication sur la date de mise à disposition (Système d'Information intégré, téléphone, rien), modalités de communication sur la charge prévisionnelle (Système d'Information intégré, téléphone, rien), etc.

\section{Grille orientée d'analyse pour le re-engineering des processus logistiques}

Une fois les caractères explicatifs obtenus, la démarche d'amélioration continue invite à développer la compréhension de ces caractères à l'aide des modèles d'entreprise présentés en partie 2.2.1.2. Au risque de retomber dans la complexité, le modèle ne doit pas chercher à décrire de façon exhaustive le système considéré. Il ne s'agit pas de modéliser pour avoir des modèles, mais de modéliser pour conduire un diagnostic et détecter les dysfonctionnements.

Dès lors, l'objectif consiste à exploiter ces modèles de représentation pour dégager des pistes d'amélioration. C'est le rôle de la phase d'analyse pour laquelle, nous l'avons vu précédemment, il n'existe pas d'outils opérationnels disponibles.

Cette partie présente une grille instrumentée permettant d'exploiter l'information contenue dans les modèles pour identifier les axes de progrès potentiels. Il s'agit également de faire la lien avec les outils de re-engineering qui seront mis en œuvre dans la phase suivante de la démarche d'amélioration continue (le traitement).

\footnotetext{
${ }^{1}$ Un caractère explicatif est une variable de l'enquête. Il existe plusieurs modalités de réponses possibles pour un même caractère explicatif. Ces modalités peuvent être hiérarchisées, à choix multiples ou numériques.
} 


\subsection{La grille instrumentée : le lien nécessaire entre le diagnostic et le traitement}

La figure suivante représente la grille d'analyse que nous proposons pour réaliser l'identification des dysfonctionnements à traiter. Nous pouvons remarquer que les lignes et les colonnes correspondent aux points de vue choisis pour la réalisation du modèle d'entreprise. Deux types d'analyses sont à considérer. D'une part, les analyses sur les points de vue, qui correspondent aux intersections de lignes et colonnes aux libellés identiques, évalueront la cohérence du modèle élaboré pour chacun des points de vue. D'autre part, les analyses inter-points de vue, correspondant aux intersections de lignes et colonnes aux libellés différents, évalueront la cohérence entre les modèles des deux points de vue.

\begin{tabular}{|c|c|c|c|c|c|}
\cline { 2 - 6 } \multicolumn{1}{c|}{} & Fonctionnel & Ressource & Organisationnel & Décisionnel & Informationnel \\
\hline Fonctionnel & 1 & 6 & 7 & 9 & \\
\hline Ressource & & 2 & 8 & 10 & \multirow{2}{*}{12} \\
\hline Organisationnel & & & 3 & 11 & \\
\hline Décisionnel & & & & 4 & \\
\hline Informationnel & & & & & \multirow{2}{*}{5} \\
\hline
\end{tabular}

\section{Figure - 4 : Grille d'analyse de la modélisation du système étudié.}

Pour chacune des intersections, l'analyse va consister en une succession de questions (check-list) sur les modèles pour identifier les dysfonctionnements. C'est ce que nous appellerons la démarche d'analyse et présentée en 4.2. La réalisation de ces analyses s'appuie sur des instruments hérités des méthodes d'amélioration des systèmes industriels. C'est ce que nous développons dans la partie 4.3.

\subsection{La démarche d'analyse pour l'identification des dysfonctionnements}

Pour nous repérer lors de la présentation de cette démarche d'analyse, nous ferons référence par un numéro à la case correspondante de la grille d'analyse. Cette numérotation correspond par ailleurs à l'ordre de réalisation de cette démarche. Nous proposons d'analyser d'abord les points de vue, puis ensuite les inter-points de vue, à chaque fois en partant du système physique, en poursuivant par le système de pilotage et en terminant par le système d'information. Analyser les points de vue avant les inter-points de vue correspond à une analyse de complexité croissante. Ensuite, l'ordre de réalisation selon les sous-systèmes physique-pilotage-information est motivé par la nécessité d'analyser d'abord le système qui doit être piloté (le 
système physique) avant de voir comment il est piloté (le système de pilotage). Pour étudier enfin comment le lien est réalisé au travers du système d'information.

Le point de vue fonctionnel (1) porte sur deux dimensions: Une première réflexion sur la valeur ajoutée associée à chaque flux doit être menée. Il s'agit d'étudier les performances (au sens des indicateurs de performance choisis) par type d'entrées et de sorties de chacune des activités de la vue fonctionnelle. Une seconde analyse est à réaliser sur la valeur ajoutée de chaque activité (étape d'un ou plusieurs flux).

L'analyse du point de vue ressources (2) correspond à la seule caractéristique intrinsèque d'une ressource : sa disponibilité. Il va s'agir ici de répondre à la question : une ressource est-elle capable de faire une tâche ou un groupe de tâches lorsqu' on le souhaite?

L'analyse du point de vue organisationnel (3) correspond à l'analyse de la cohérence du modèle organisationnel, celui-ci pouvant être défini comme un ensemble d'unités organisationnelles inter-reliées. Par exemple, une unité organisationnelle dépendant à la fois de deux unités organisationnelles supérieures sans coordination entre les deux, n'est pas un système organisationnel cohérent.

L'analyse du point de vue décisionnel (4) correspond à la cohérence du modèle décisionnel formulé. Il s'agit ici de détecter les éventuels manques en termes de cadre de décision, en termes de disponibilité et d'exhaustivité d'information pour la prise de décision.

Enfin, le dernier point de vue à analyser est informationnel (5). Nous considérons l'information comme un support aux autres points de vue. L'analyse peut alors être menée sur:

- la fiabilité de l'information ;

- la cohérence entre les différentes occurrences d'une même information ;

- et la disponibilité de l'information (actualisation et mise à disposition).

La notion de fiabilité de l'information renvoie au fait qu'une information donnée a un sens en accord avec celui qu'elle devrait avoir. Nous pouvons prendre l'exemple d'un stock ayant une certaine valeur dans le système d'information, alors que l'emplacement de stockage est vide. La cohérence entre les différentes occurrences fait référence à la problématique d'unicité de l'information afin d'éviter les incohérences. Par exemple, l'information sur un stock est présente dans deux systèmes d'information différents (gestion de stock et comptable par exemple) et la valeur n'est pas la même. Enfin, la disponibilité de l'information est liée à la problématique de sa mise à disposition (récupérer l'information et la transférer) ainsi que de son actualisation. L'information sur un niveau de stock n'a aucune valeur pour un planificateur de production si elle date de plusieurs jours ou si il ne peut pas $\mathrm{y}$ avoir accès aisément. 
L'analyse croisée des points de vues fonctionnel/ressource (6) recouvre plusieurs questions. Premièrement, nous allons considérer l'état statique du système en analysant les compétences (dans le cas des ressources humaines) ou les aptitudes (dans le cas des ressources techniques : machines et logiciels) en fonction de celles qui sont requises par les différentes activités les traversant. Deuxièmement, nous nous intéressons au caractère dynamique du système en déterminant si les ressources sont flexibles et réactives par rapport aux flux qui les traversent. Une ressource est dite flexible si elle peut effectuer plusieurs types d'activités différentes. Une ressource est dite réactive si elle peut passer d'un type d'activité à une autre de façon rapide. Troisièmement, l'organisation des flux entre les différentes ressources estelle réalisée correctement ? Il s'agit là d'analyser les différentes manières d'organiser des processus physiques et de choisir celle qui est la plus adaptée. Enfin, la dernière analyse à mener concerne l'adaptation des flux aux ressources requises. En effet, un inducteur de performance peut être de (re)concevoir les produits soit en les standardisant, modularisant... soit en les adaptant au processus de production.

L'analyse fonctionnel/organisationnel (7) pose le problème de la cohérence entre les processus fonctionnels et les unités organisationnelles traversées. Tout d'abord, il doit être sain qu'une unité organisationnelle soit mise en jeu dans un processus fonctionnel donné. En outre, lorsqu'un processus fonctionnel traverse plusieurs unités organisationnelles, les interfaces sont-elles claires et affichées ?

L'analyse ressources/organisationnel (8) pose la question de l'adéquation de la charge de gestion de l'unité organisationnelle avec la capacité disponible pour effectuer cette tâche de gestion. En effet, certaines ressources sont liées pour tout ou partie de leur charge de la gestion d'un système industriel (flux, unité organisationnelle, etc.). Une autre analyse pertinente concerne la cohérence de l'affectation des ressources avec la décomposition organisationnelle. On peut illustrer cette analyse par le cas d'une usine qui aurait plusieurs ateliers et dont l'un des ateliers serait piloté par une autre usine.

L'analyse fonctionnel/décisionnel (9) permet de s'assurer que les centres de décision nécessaires et suffisants existent pour gérer l'ensemble des flux et des activités d'un modèle fonctionnel, pour tous les niveaux de prise de décision adaptée (opérationnel, tactique, stratégique).

Nous retrouvons une question similaire lors de l'analyse ressource/décisionnel (10) afin de vérifier que les centres de décisions nécessaires et suffisants existent pour gérer l'ensemble des ressources. Cette analyse touche également à l'adéquation charge/capacité des ressources et le pilotage de ce ratio par le centre de décision adéquat. Enfin, nous devons également nous intéresser à la cohérence des centres de décisions du point de vue des ressources gérées. En effet, il est important que les ressources pilotées par un centre de décision soient du même niveau dans la décomposition hiérarchique des ressources. Nous pouvons imaginer la difficulté d'un centre de décision à gérer simultanément la planification d'un ensemble d'unités de production d'un réseau logistique et l'ordonnancement d'un atelier 
regroupant quelques machines et quelques personnes. Il est sain qu'un centre de décision pilote l'ensemble des ressources de son périmètre correspondant à un niveau donné de la hiérarchie des ressources.

L'analyse décisionnel/organisationnel (11) requiert une vérification de la cohérence entre les centres de décisions et les unités organisationnelles. Il est important de pouvoir associer à chaque unité organisationnelle un ensemble de centres de décision précis. Lors de cette analyse, il est important également de vérifier qu'une unité organisationnelle n'ait pas la responsabilité d'un demi centre de décision (ne pouvant agir que sur une partie des inducteurs de performance) et qu'une autre unité organisationnelle ait l'autre moitié.

Enfin, pour terminer le parcours de cette grille, nous devons procéder à l'analyse du point de vue informationnel (12) avec l'ensemble des autres points de vue. L'information étant un support pour les autres points de vue, l'analyse va porter ici sur l'identification des informations nécessaires.

Une fois ces analyses menées, des pistes d'amélioration peuvent être identifiées. Ces pistes doivent être alors évaluées en termes d'accessibilité et d'enjeux, afin de savoir si la mise en oeuvre des actions appropriées sera réalisée.

L'analyse aurait pu être poussée jusqu'à s'interroger sur les intersections entre trois ou quatre points de vue. Cela rajoute bien évidemment de la complexité et au vu de notre expérience, cela n'est pas nécessaire. Notre proposition de grille s'arrête donc à l'analyse des interactions entre deux points de vues.

\subsection{Instrumentation de l'analyse}

Nous proposons maintenant d'instrumenter la grille que nous venons d'exposer (cf. Figure 5). Il s'agit de lister les outils et méthodes nécessaires à la réalisation de l'étape d'analyse associée à chaque point de vue et inter-points de vue. Il s'agit aussi de mettre en perspective les outils et méthodes susceptibles de faciliter le traitement ultérieure des différentes typologies de problèmes pouvant exister au niveau de chaque point de vue ou inter-points de vue. 


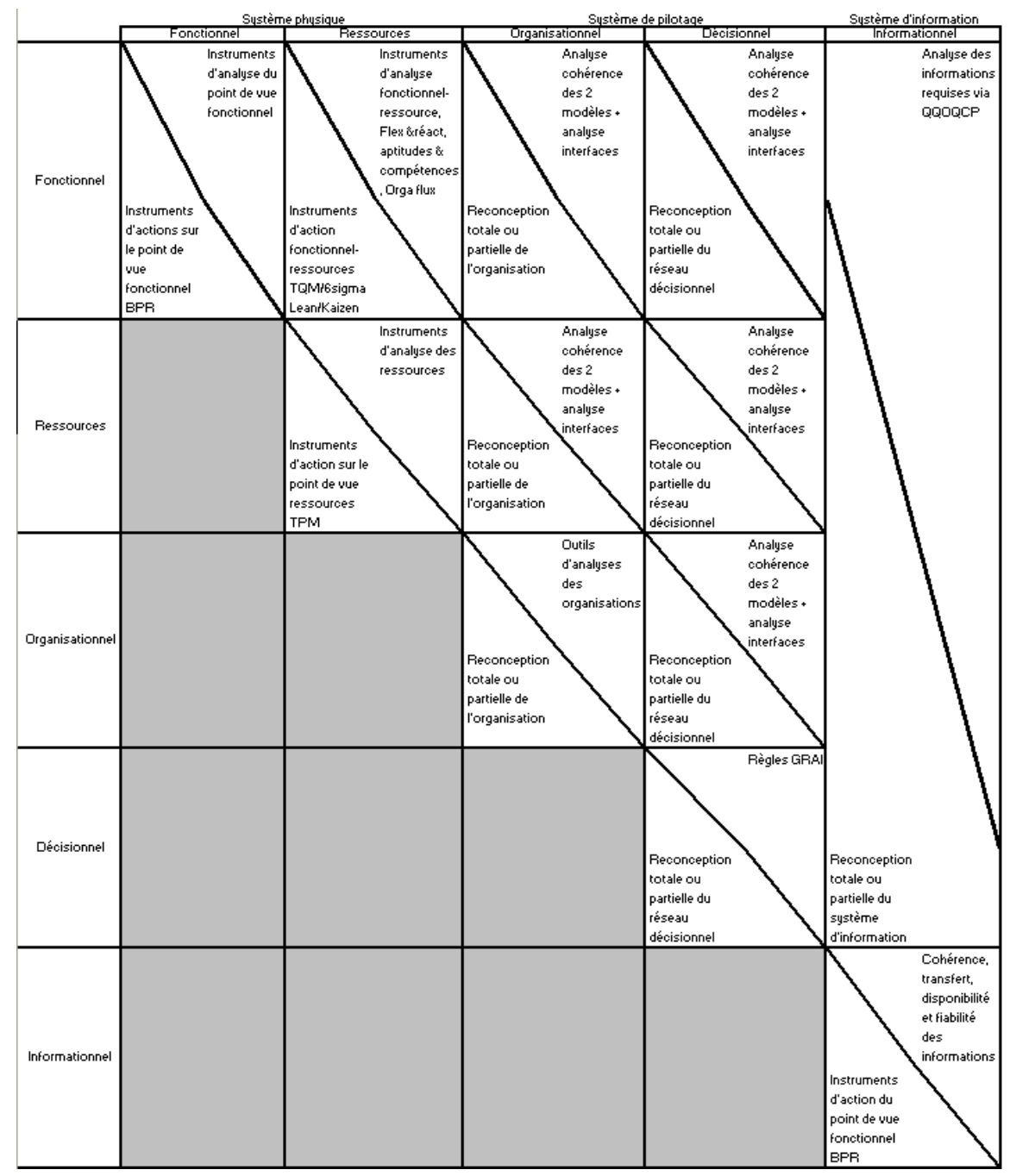

Figure - 5 : Instrumentation de la grille pour l'analyse et méthodes d'action correspondante.

Chaque case (ou groupe de cases en cas de regroupement) sera instrumentée pour l'analyse d'une part et pour l'action d'autre part. Historiquement, c'est principalement le système physique qui a fait l'objet de toutes les attentions, puisqu'il constitue le fondement même de l'activité d'un système industriel. Ainsi, comme nous l'avons vu dans l'état de l'art présenté au chapitre précédent, plusieurs démarches et de nombreux instruments ont été créés relativement aux points de vue fonctionnel et ressource. Plus récemment, les systèmes de décision et d'information ont été travaillés afin d'améliorer la performance globale du système. Les outils sont 
donc moins largement diffusés du fait de cette relative jeunesse. Nous allons reprendre la grille case par case pour identifier les instruments d'analyse et d'action.

Concernant le point de vue fonctionnel (1), les instruments d'analyse et d'action sont ceux qui ont été évoqués en partie 2.3 (analyse de la valeur, process mapping, cahier des charges fonctionnels, etc.). Nous pouvons compléter cette instrumentation par la démarche BPR qui a vocation à être fortement axée sur le point de vue fonctionnel. L'un des principes du BPR consiste par exemple à paralléliser au maximum les activités qui peuvent l'être.

Pour le point de vue ressource (2) la partie 2.3 présente également les principaux instruments associés (5M, arêtes de poisson, capabilité, etc.). Nous y ajoutons la démarche TPM en raison de sa forte connexion avec les préoccupations de cette case de la grille. On pourra aussi ici se baser sur des mesures de Taux de Rendement Synthétique.

Le point de vue organisationnel (3) est instrumenté grâce au domaine de la gestion des organisations. Cependant, nous estimons nos compétences limitées sur ces aspects et donc notre proposition est certainement partielle sur ce point. Nous instrumentons néanmoins l'analyse menée à cette case par une réflexion sur le lien entre les différentes unités organisationnelles de l'organigramme, leur mode de management et comment en est assurée la direction. La mise en pratique industrielle se fera fréquemment avec des spécialistes de ces questions que l'on retrouve le plus souvent dans les fonctions de Ressources Humaines. En revanche, la mise en oeuvre se fera soit par une re-conception partielle de la structure organisationnelle (et de son modèle, l'organigramme), soit par une re-conception totale.

Le point de vue décisionnel (4) est instrumenté nativement par le modèle GRAI. En effet, celui-ci dispose d'un ensemble de règles pour s'assurer de la cohérence intrinsèque de la grille GRAI. Ce sont donc ces onze règles que nous proposons d'utiliser pour mener cette analyse. La définition des actions correctives se fera - de même que précédemment - par une re-conception partielle ou totale de la structure décisionnelle.

Le point de vue informationnel (5) est instrumenté au travers de règles établies par les modèles dédiés à l'analyse des éléments informationnels. Ainsi, un certain nombre de règles sont sous-jacentes au modèle Entités- Associations. Par exemple, un identifiant existe pour chaque entité. Cependant, la vérification de la cohérence du modèle dépend du sens donné. Des outils logiciels existent pour valider la cohérence d'un modèle, pour une sémantique définie. Nous pouvons en outre utiliser ici une approche BPR pour améliorer la pertinence de la récupération de l'information à la source, son unicité ou encore le traitement de l'information par le collecteur.

Pour l'inter-point de vue fonctionnel-ressources (6), les instruments disponibles pour l'analyse sont en partie listés au paragraphe 2.3 (AMDEC, Audit, Benchmarking, Pareto, etc.). Nous pouvons compléter cette liste en y ajoutant 
l'analyse de l'adéquation entre les compétences/aptitudes des ressources et celles requises par les flux les traversant (en utilisant des modèles de Simulation par exemple). En outre, l'analyse de la flexibilité et de la réactivité des ressources en fonction des flux les traversant peut être menée. Bien qu'il n'y ait pas d'instrument à proprement parler, il suffit de vérifier si chaque type de ressource est flexible et réactif en fonction des contraintes de flux qui les traversent. Le choix entre les différentes démarches possibles d'amélioration (TQM, 6 Sigma, Lean / Kaïzen, etc.) pourra se faire en lien avec le besoin exprimé (est-ce plutôt un problème de ressources ou plutôt un problème de flux ?), en fonction du type d'approche souhaitée (changement plutôt en douceur ou plutôt de façon rapide) ou encore en fonction de l'organisation générale de l'entreprise et de sa culture, comme le propose [Siebenborn, 2005].

Pour les inter-points de vue organisationnel-fonctionnel et organisationnelressources (7 et 8), il n'existe pas d'instruments a priori pour les analyser. Nous proposons une confrontation des deux modèles entre eux. Pour l'inter-point de vue organisationnel-ressources (8), la tâche de gestion de l'unité organisationnelle peut être appréhendée au moyen d'une classique analyse charge/capacité. L'instrumentation de l'action sur ces deux cases se fait comme pour la case 3 par une re-conception totale ou partielle de l'organisation. En ce qui concerne les actions liées à une mauvaise adéquation charge/capacité pour la gestion d'une unité organisationnelle, des actions peuvent être menées, comme la révision de la charge de gestion ou encore la modification de la capacité par un ajout de ressources. Les analyses sur les cases 7 et 8 ne peuvent être entreprises que si les points de vue fonctionnels et ressources sont stabilisés, ce qui confirme la logique d'investigation proposée au début du chapitre.

Pour les inter-points de vue décisionnel-fonctionnel, décisionnel-ressources et décisionnel-organisationnel $(9,10$ et 11$)$, nous retrouvons les mêmes instruments d'analyse que ceux présentés précédemment pour les cases 7 et $8:$ confrontation des deux modèles et des interfaces, et re-conception totale ou partielle de réseau décisionnel.

Enfin, pour les inter-points de vue liés au point de vue informationnel (12), l'instrumentation de l'analyse est faite avec le QQOQCP (Qui ? Quand ? Où ? Quoi ? Comment ? Pourquoi ?). En effet, cet instrument permet d'analyser les besoins en information des autres points de vue : «Qui utilise quelle l'information ? Où ? Comment ? Quand ? Pourquoi ? ».L'instrumentation de l'action va se traduire par une re-conception totale ou partielle du système d'information.

À l'issue de l'utilisation de cette grille d'analyse, des dysfonctionnements et axes d'amélioration ont dû apparaître. Des actions de traitement doivent alors être définies et des cibles doivent être proposées. 


\subsection{Application de la grille Analyse / Actions}

Nous présentons l'application de cette grille au cas des laboratoires de contrôle qualité de la chaîne logistique présentée en partie 3.3. Nous nous focalisons principalement sur la partie décisionnelle du système. Nous détaillons une analyse et les actions concernant ce point de vue et l'inter-point de vue décisionnel-ressources. Pour plus de développements sur les applications de la grille le lecteur pourra se référer à [Dupuy, 2005].

\subsubsection{Phase de modélisation}

A noter que cette étape s'appuie sur les éléments collectés, à l'aide du référentiel de diagnostic des chaînes logistiques, lors de la phase d'observation.

La modélisation GRAI permet de représenter la partie périodique du système de conduite et d'en évaluer la pertinence ainsi que la cohérence par rapport à l'environnement. Dans la modélisation, nous avons fait le choix de nous intéresser exclusivement aux deux entités que sont la production et le contrôle qualité, étant donné que nous nous intéressons principalement au temps de cycle des analyses de routine, donc la relation client/fournisseur entre la production, les approvisionnements et le contrôle.

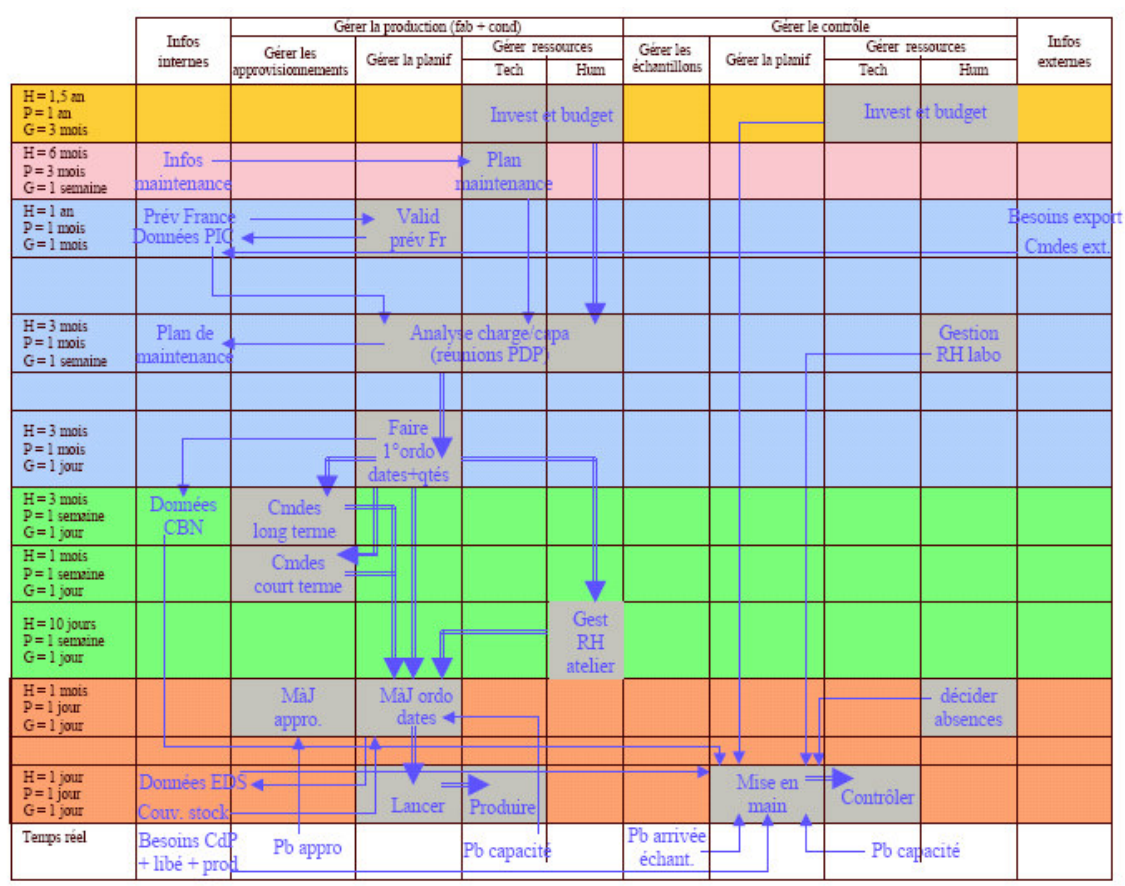

Figure - 6 : Grille GRAI. 


\subsubsection{Phase d'analyse des modèles}

\subsubsection{Décisionnel}

La colonne «Gérer le contrôle » de la grille GRAI (cf. figure 3.3) est quasiment vide : le seul pilotage est un pilotage temps réel de la file d'attente de mise en main des analyses présentes au laboratoire. La seule activité de planification est liée à la gestion des ressources humaines sans évaluation précise de la charge. Ainsi, notre premier constat est le manque de centres de décision au niveau du contrôle qualité afin de pouvoir piloter correctement le système. De ce fait, il semble pertinent de créer un centre de décision moyen terme, afin de gérer de façon prévisionnelle l'adéquation charge/capacité. Ensuite, le deuxième constat est la présence d'un réordonnancement quotidien de l'usine, du fait de la confrontation quotidienne des plannings de production avec les couvertures de stock liées aux ventes. Cette constatation met en évidence la nécessité d'une analyse de la flexibilité et de la réactivité des différentes ressources des laboratoires pour suivre les réordonnancements quotidiens de l'ensemble de la production. Enfin, le troisième constat est le positionnement du centre de décision du contrôle qualité appelé «Mise en main ». Celui-ci opère sans vision prévisionnelle suffisante, étant donné que l'horizon est égal à la période ( $\mathrm{H}=\mathrm{P}=1$ jour), mais aussi que l'horizon est inférieur au temps de cycle de l'opération qu'il gère $(\mathrm{H}<\mathrm{d}$, $\mathrm{d}$ est de 3 jours en moyenne). On se rend ainsi compte que ce centre de décision a un positionnement en temps réel par rapport au système opérant et qu'il est obligé de générer une longue file d'attente pour pouvoir gérer ses activités. Cependant, ce positionnement n'est pas incohérent, étant donné qu'il doit permettre de piloter finement l'activité : il faut donc chercher à augmenter son horizon. Cette constatation renforce les deux premières conclusions disant qu'il faut un centre de décision moyen terme pour le pilotage de l'équilibre charge/capacité de façon prévisionnelle, mais aussi mener une analyse complémentaire pour vérifier la flexibilité et la réactivité des différentes ressources.

\subsubsection{Ressources-Décisionnel}

Les déterminations par chromatographie (utilisant des appareils appelés HPLC ou CPG qui nécessitent un temps de préparation très long en regard du temps opératoire) ont un impact important sur la performance du système en termes de délai, ce qui nécessite un pilotage particulier de ce type de ressource avec un système de décision adapté (aide à la décision nécessaire étant donné la complexité du problème), ainsi qu'une coordination avec le pilotage des autres ressources du laboratoire de contrôle qualité. Un centre de décision spécifique d'un type de ressources est donc nécessaire. 


\subsubsection{Traitement des dysfonctionnements mis en lumière}

\subsubsection{Décisionnel}

La première action entreprise a été d'obtenir une amélioration de la visibilité des flux de contrôle qualité. Pour ce faire, un tableau mural a été installé dans lequel des fiches en $\mathrm{T}$ viennent représenter l'activité et l'étape à laquelle en est l'analyse dans le processus. Cet outil permet à la fois une visibilité aisée sur l'activité des laboratoires de contrôle, un partage de l'information avec les clients internes et une source d'information permettant de dresser hebdomadairement des tableaux de bord d'activité.

Concernant le très court terme, les laboratoires ont besoin de savoir quelles sont les analyses pour lesquelles le résultat est attendu : une expression des besoins clients à court terme. Cependant, ces informations ne sont pas suffisantes pour être en mesure d'effectuer un lancement des analyses étant donné que l'horizon de visibilité est trop réduit. Pour le domaine du court terme et notamment de l'aide au lancement des analyses, les laboratoires ont besoin d'éléments indicatifs pouvant être obtenus par une information du type : (date de besoin) - (durée du cycle); obtenus via des états issus de requêtes dans l'ERP. Une évolution de ces outils simples a été menée en les intégrant à une transaction de gestion du lancement. L'agent de maîtrise chargé de l'affectation des analyses aux techniciens aura ainsi la liste de travail triée par priorité qui lui apporte une aide à la décision sur l'analyse à lancer ainsi que des informations relatives aux compétences des techniciens, à la charge de travail induite pour le technicien par l'analyse affectée, etc.

Pour l'aspect moyen terme, il faut se souvenir que la durée de formation d'un technicien durant généralement de un à trois mois, le démarrage d'un technicien recruté est nécessaire avant la période de charge à laquelle il doit être opérationnel. Ainsi, une démarche d'analyse du taux de charge des différentes ressources doit être mise en oeuvre.

\subsubsection{Ressources-Décisionnel}

En ce qui concerne le centre de décision sur la gestion des ressources chromatographiques, avant de pouvoir passer à la mise en ouvre opérationnelle il faut pouvoir être en mesure d'apporter un outil d'aide à la décision permettant de gérer la complexité de ce problème. Il s'agit alors d'un problème d'ordonnancement nouveau étudié dans [Dupuy et al. 2004], [Dupuy et al. 2005] et [Dupuy 2005].

\section{Conclusion et perspectives}

Les éléments que nous venons de présenter s'appliquent lorsque les causes de dysfonctionnement ne sont pas évidentes (ce qui est généralement le cas des processus logistiques par nature transverse). Nous avons identifié dans ce papier 
deux points durs en termes d'outils supports à la mise en œuvre d'une démarche d'amélioration continue de processus logistiques.

D'abord des lacunes en termes de méthodologies d'enquêtes. Les outils classiques existants ne permettant pas réellement d'appréhender la complexité des chaînes logistiques. Nous avons dès lors proposé un référentiel de diagnostic des chaînes logistiques et de leurs composantes coopératives. L'objet de ce référentiel est de structurer la démarche de diagnostic et par-là de préparer la phase de modélisation dans le cadre d'une démarche d'amélioration continue des processus logistiques. Il permet de structurer la prise de connaissance des acteurs et de leurs interactions. Même sans descendre au niveau de leur formulation la structure de notre référentiel laisse présager un très grand nombre de questions (environ un millier !). Il est évident qu'une telle combinatoire rendrait quasiment impossible la mise en œuvre. C'est pourquoi il faut préciser qu'en fonction du but de la mission réalisée, l'étude de toutes les fonctions de la chaîne ne sera sans doute pas utile (ou en tout cas peut-être pas avec le même niveau de finesse). Il est également envisageable d'utiliser le référentiel dans le cadre d'une étude qui ne porterait que sur la « coordination du $S C M$ » (fonctionnement local) ou que sur «l'intégration du $S C M »$ (fonctionnement des ententes). C'est donc en effectuant des coupes en fonction de ses objectifs que l'utilisateur pourra exploiter réellement un tel référentiel. L'exploitation du référentiel d'analyse des systèmes logistiques conduit à l'obtention d'une grande quantité d'informations. Mais la profusion n'étant pas forcément synonyme de richesse, nous proposons dans [Lauras, 2004] d'utiliser des méthodes d'analyse de données afin de pouvoir exploiter pratiquement les résultats obtenues dans le cadre d'une démarche d'amélioration continue des chaînes logistiques et de leurs composantes coopératives.

Ensuite, nous avons établi un constat de carence en termes de méthodes d'analyse permettant de passer de l'étape de modélisation de la démarche d'amélioration continue à l'étape de traitement. Nous avons donc imaginé un outil permettant de définir quels sont les points à améliorer et quels sont les supports pour mettre en oeuvre cette amélioration. Concrètement, nous avons proposé - pour les systèmes industriels - une grille d'analyse/action instrumentée partant des modèles d'entreprise avec ses différents points de vue pour identifier dans un même temps, les dysfonctionnements et les orientations à exploiter pour les traiter. La check-list est, d'une part, basée sur la structure des modèles utilisés et leur confrontation. D'autre part, cette check-list hérite de l'expérience industrielle des partenaires du projet pour pouvoir critiquer les modèles. En revanche, si des modèles plus détaillés sont utilisés ou si un nouveau point de vue est à développer (environnemental par exemple), alors elle atteint ses limites. Des perspectives possibles de travail concernent enfin l'établissement de liens formels entre les outils que nous venons de décrire et les méthodes d'évaluation de performance. Il s'agit notamment d'étudier le lien entre le type de performance souhaitée et les points de vues et inter-points de vues à analyser d'autre part. Bien qu'il existe de réelles problématiques quant à 
l'utilisation de ces méthodes dans le cadre de processus logistiques [Lauras, 2005], nous n'avons pu les aborder dans le cadre de ce papier.

\section{Bibliographie}

Arnold T., Chapman S.N., Introduction to Material Management, Prentice Hall, 4ème Edition, 2001

ASLOG, Référentiel de la performance logistique, Editions ASLOG, 2003

Balantzian G., Les systèmes d'information : arts et pratiques ; la vision globale, Editions d'Organisation, 2002

Berrah L., Haurat A., Une stratégie de mise en place d'indicateurs de performance pour le pilotage de processus de production, MOSIM, Rouen, 1997

Berrah, L., Clivillé, V., Harzallah, M., Haurat, A., et Vernadat, F., PETRA : un guide méthodologique pour une démarche de réorganisation industrielle. Université de Savoie - Université de Metz, http ://www.listic.univ-savoie.fr/projet/petra/, 2001

Burlat P., Marcon E., Sénéchal O., Dupas R., Berrah L., Concepts pour la performance des systèmes de production, Chapitre d'ouvrage «Evaluation des performances des systèmes de production » sous la direction de C. Tahon, Edition Hermès, p.49-80, 2003

Burlat P., Modélisation et pilotage des organisations en réseau, Habilitation à Diriger les Recherches, Ecoles des Mines de Saint-Etienne, 2002

Doumeignts G., Vallespir B., Modélisation d'entreprise : vers le système d'information, Actes de l'école de modélisation d'entreprise, Ecole des Mines d'Alès, 2004

Dupuy M., Contributions à l'analyse des systèmes industriels et aux problèmes d'ordonnancement à machines parallèles flexibles, Thèse de doctorat de l'INPT, 2005

Dupuy M., Lamothe J., Gaborit P., et Dupont L. Ordonnancement à machines parallèles à usages multiples avec ressources secondaires, temps de préparation et calendrier de disponibilité. In $5^{\circ}$ conférence francophone de MOdélisation et SIMulation, 2004

Dupuy M., Lamothe J., Gaborit P., et Dupont L., Efficient neighbors in a simulated annealing problem to optimize the lead time in a parallel multipurpose machine scheduling problem with setup and calendar constraints. In International conference on Industrial Engineering and Systems Management (IESM), 2005

Eckes G., Objectif Six Sigma: Révolution dans la Qualité, Edition Village Mondial Pearson Education France, 286 p., 2001

Eymery P., La logistique de l'entreprise : Supply Chain Management, Edition Hermes, 217 p., 1997

Stadtler H., C. Kilger, Supply Chain Management and Advanced Planning, Edition SpringerVerlag, 2000

Hammer M., Champy J., Le re-engineering : réinventer l'entreprise pour une amélioration spectaculaire de ses performances, Editions Dunod, 1993

Kearney A.T., Management approach to supply chain integration, Rapport aux membres de l'équipe de recherche A.T. Kearney, Chicago, 1994

Kettinger, W., Teng, W. J., et Guha, S., Business process change, a study of methodologies, techniques and tools. Management Information Systems Quarterly, p. 55-80, 1997

Lauras M., Pingaud H., Lamothe J., Proposition de référentiel de diagnostic des chaînes logistiques et de leurs composantes coopératives, Gème Congrès International de Génie Industriel, Besançon, 2005

Lauras M., Méthodes de diagnostic et d'évaluation de performance pour la gestion de chaînes logistiques, Thèse de doctorat de l'INP Toulouse, 2004 
Lauras M., Parrod N., Telle O., Proposition de référentiel pour la notion d'entente industrielle : trois approches dans le domaine de la gestion des chaînes logistique, Revue Française Gestion Industrielle, Vol. 22, $\mathrm{N}^{\circ}$ 4, 2003

Tayur S., Ganeshan R., Magazine M., Quantitative models for supply chain management, Kluwer Academic Publishers, 2000

Mentzer J.T., Dewitt W., Keeber J.S., Min S., Nix N.W., Smith C.D., Z.G. Zacharia, Defining the supply chain management, Journal of Business Logistics, $\mathrm{Vol} 22, \mathrm{~N}^{\circ} 2,2001$

Morley C., La modélisation des processus : typologie et proposition utilisant UML, Assises ADELI, 2002

Normes Qualité ISO 9000, Editions AFNOR, 2000

Pingaud H., L'hôte V., Logistique et technologies de l'information et de la communication, Editions WEKA, Broché, 115 p., 2004

Pingaud, H., Modélisation d'entreprise. Cours Master Productique de l'Université Paul Sabatier de Toulouse, 2005

Poirier C.C., Reiter S.E., Revoir le partenariat d'entreprise, La Supply Chain, Editeurs : C.C. Poirier, S.E. Reiter, Dunod, 285 p., 2001

Rouquet A., Global EVALOG, Dossier GALIA, 2004

Sardas J.-C., Erschler J., de Terssac G., Coopération et organisation de l'action collective, PROSPER, 2002

Supply Chain Council, Supply Chain Operations reference-models - SCOR version 4.0, Edition Supply Chain Council, 214 p., 2000

Villarreal C., Pingaud H., Gourc D., Rapport intermédiaire de recherche sur l'utilisation de la théorie des jeux pour le pilotage des réseaux d'entreprise, Centre Génie Industriel, EMAC, 2004 